\title{
Thermal imaging as a modern form of pyrometry
}

\author{
U. Kienitz \\ Optris GmbH, Berlin, Germany \\ Correspondence to: U. Kienitz (ulrich.kienitz@optris.de)
}

Received: 29 January 2014 - Revised: 6 August 2014 - Accepted: 18 August 2014 - Published: 29 October 2014

\begin{abstract}
Pyrometers and thermography cameras used to be characterized by different specifications and technical definitions. After an analysis of the market situation and the physical basics, the following article describes common methods to determine optical and thermal key parameters. Based on this, aspects of future sensor developments and certain applications of infrared (IR) cameras are discussed.

(This article was first published in the journal tm-Technisches Messen, Vol. 81, No. 3, 2014, a volume which is dedicated to the memory of Prof. Dr.-Ing. habil. Ludwig Walther.)
\end{abstract}

\section{Market and trends}

Military and security applications will continue to be the main markets for infrared (IR) thermometry. The night combat capabilities provided by this technology have completely changed combatants' attacking strategies in conflicts like those in the Middle East. Another use for target acquisition devices which are immune to adverse weather and light conditions can be found in drone applications (FLIR Corporation, 2013). In the civilian sector, however, the current use of thermal imaging cameras in motor vehicles - with an installation rate below $1 \%$ - is still far short of initial expectations (YOLE Developement, 2010). But the market for thermal imaging measurement systems has nevertheless greatly expanded. The sole driver of growth has been the price developments of these mainly hand-held IR cameras. More efficient production methods, particularly the wafer-level vacuum packing for the widely used thin-layer bolometers, have significantly reduced product costs. Hand-held units have traditionally been used for electrical and mechanical maintenance applications, as well as for detecting thermal leakages in buildings. Stationary IR cameras, when used for test and measurement and in automation applications, have benefited from the availability of inexpensive IR focal plane arrays.

\section{Imaging devices are displacing pyrometers}

Point-measuring radiation thermometers have been preferred over cameras for industrial measurement applications because of their ruggedness, ease of use and ease of integration. The advantages that cameras offer in locating thermal inhomogeneities have been tempered by their significantly higher costs. Ten years ago, hand-held pyrometers maintained a predominant market share for maintenance applications. But since then, the market has shifted such that more and more imaging devices are being used. This shift away from the use of pyrometers is also occurring for the temperature measurement systems used in stationary applications by machine and equipment builders. New systems often use cameras instead of pyrometers because the cameras offer better variability and programmability of measurement positions.

The technical properties of cameras and pyrometers differ significantly. The VDI 3511 standard describes basic parameters of IR thermometers (VDI/VDE 3511, 2004). Similar definitions for thermal cameras only exist currently in draft form (VDI/VDE 3511, 2010). The key measurement criteria for both types of devices are compared below. We are limiting our focus here on economically relevant measurement systems used to detect objects within the standard ambient temperature range. For reasons of physical radiation, such devices usually operate in the $8-14 \mu \mathrm{m}$ spectrum. For their detectors, the IR cameras use uncooled thermal micro-bolometer arrays that range from $80 \times 80$ pixels to $1024 \times 768$ pixels, with pixel sizes ranging from $50 \times 50$ to $12 \times 12 \mu \mathrm{m}$ (Durand et al., 2011). Such focal plane arrays (FPAs) require a mechanical shutter for offset compensation. This is necessary due to the basic principle of 


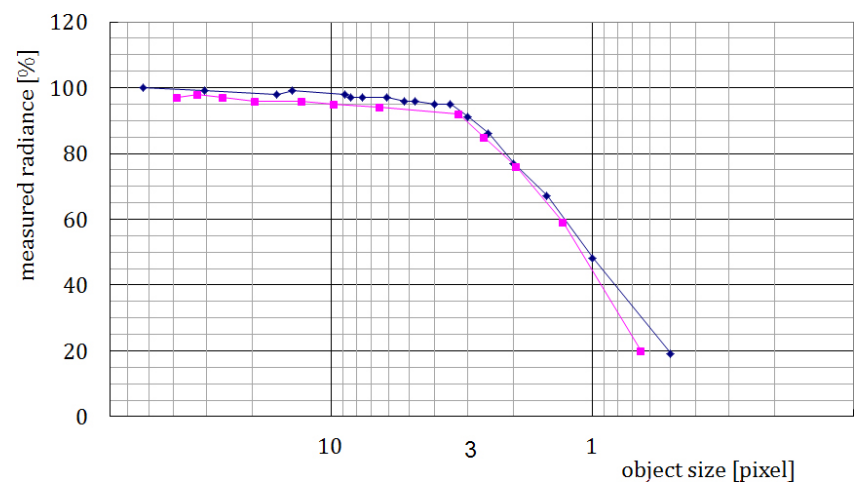

Figure 1. Geometric resolution of two commercially available IR cameras with an FOV of approximately $20^{\circ}$.

bolometric resistance bridges and the non-negligible drift in the amplification and serialization electronics. Because of their constant light sensitivity, thermopiles with effective sensor diameters ranging from 0.4 to $2 \mathrm{~mm}$ are normally used for radiation thermometers (Datasheet Heimann Sensor $\mathrm{GmbH}, 2013$; Datasheet Micro-Hybrid Electronic GmbH, 2013). Under similar performance parameters, pyro-electric sensors require chopper motors for light modulation. Based on a $63 \%$ step response, the time constant for thermal sensors is in a range of $5-150 \mathrm{~ms}$. Pyrometers with uncooled extended InGaAs (indium gallium arsenide) photodiodes are an exception; they have time constants well below $1 \mathrm{~ms}$, but they only detect object temperatures starting at $50^{\circ} \mathrm{C}$. In addition, such photodiodes operate in the $1.6-2.3 \mu \mathrm{m}$ range, which makes them sensitive to daylight.

\section{Parameterization of IR cameras and radiation thermometers}

\subsection{Optical resolution}

Based on the specific detectivity $\left(D^{*}\right)$ of the core component of the IR measuring device - the thermal detector or the photodiode - its performance can be calculated when the type of optics used is also taken into consideration. Thus the optical resolution can be optimized at the expense of the thermal resolution across a wide range. The key parameter for a pyrometer is the distance ratio $\left(D_{/ S}\right.$ geo $)$ :

$D_{/ S \text { geo }}=\frac{a_{\mathrm{m}}}{d_{\mathrm{m}}}$.

The normal values for this ratio between measuring distance $\left(a_{\mathrm{m}}\right)$ and spot diameter $\left(d_{\mathrm{m}}\right)$ are $0.5-150$ for devices measuring from -50 to $150^{\circ} \mathrm{C}$. For higher object temperatures beginning from $150^{\circ} \mathrm{C}$, pyrometers can be designed with distance ratios from 20 to 300, which are usually operating at shorter wavelengths. When determining the distance ratio $\left(D_{/ S}\right.$ meas $)$, the relevant factor at a specific measuring distance is the diameter of a black body at which the radiation

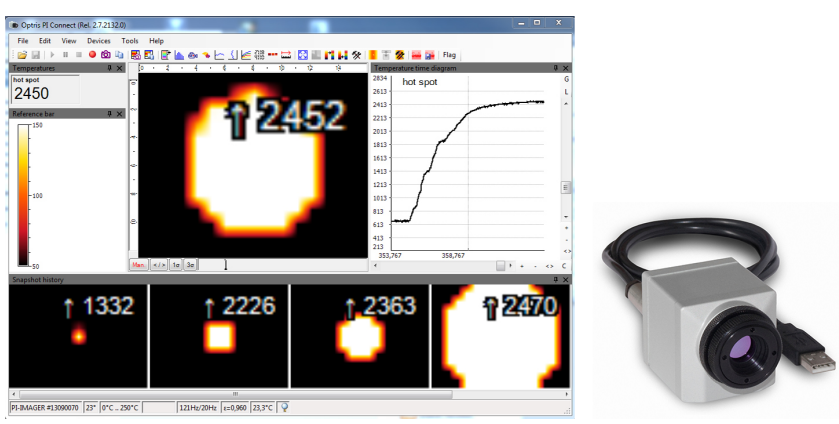

Figure 2. Radiation proportional values for the central pixel at illuminations of (a) $1 \times 1$, (b) $3 \times 3$ and (c) $5 \times 5$, and (d) a sufficient number of pixels (infrared camera PI160 from Optris; $23^{\circ}$ lens; values from a 14 bit video AD converter).

signal has decreased by $10 \%$ compared to the signal of a sufficiently large black body. This definition takes into consideration the spherical and chromatic aberration of the optics, the effective detector area and the scattering effects of the optical channel. Taking into account solely the main parameters of the optics - their effective aperture respectively lens diameter $\left(d_{L}\right)$ and their f-number $(F)$ - according to Product brochure Optris $\mathrm{GmbH}$ (2013) a geometric distance ratio can be calculated using the sensor area $\left(A_{\mathrm{s}}\right)$ :

$D_{/ S \text { geo }}=\frac{F d_{L}}{\sqrt{A_{\mathrm{S}}}}$.

For large sensor dimensions, compared to the wave length and coated IR glass lens systems, this geometric target distance corresponds in good approximation to the measured target distance. For imaging devices, single-pixelbased fields of view IFOV $_{\text {geo }}$ are used instead of distance ratios. This value is calculated from the field of view (FOV) and the number of associated horizontal and vertical pixels $\left(p_{\#)}\right)$

$\mathrm{IFOV}_{\mathrm{geo}}=\frac{\mathrm{FOV}}{p_{\#}}$.

Values ranging from 0.3 to $15 \mathrm{mrad}$ are typical. The following formula is used to calculate the distance ratio from this parameter:

$D_{/ S \text { geo }}=\frac{1}{\text { IFOV }_{\text {geo }}}$.

Figures 1 and 2 show the dependence of the displayed radiance on the object dimensions shown as a multiple of the pixel size for commercially available IR cameras. Since the dimensions of the individual pixels are already similar to the wavelength being detected, the resolution of the optical system is limited.

So the object size must be at minimum three pixels, corresponding to a three-time $\mathrm{IFOV}_{\text {geo }}$ to specify a distance ratio $\left(D_{/ S}\right.$ meas $)$ with a sufficient $90 \%$ radiation signal. This 


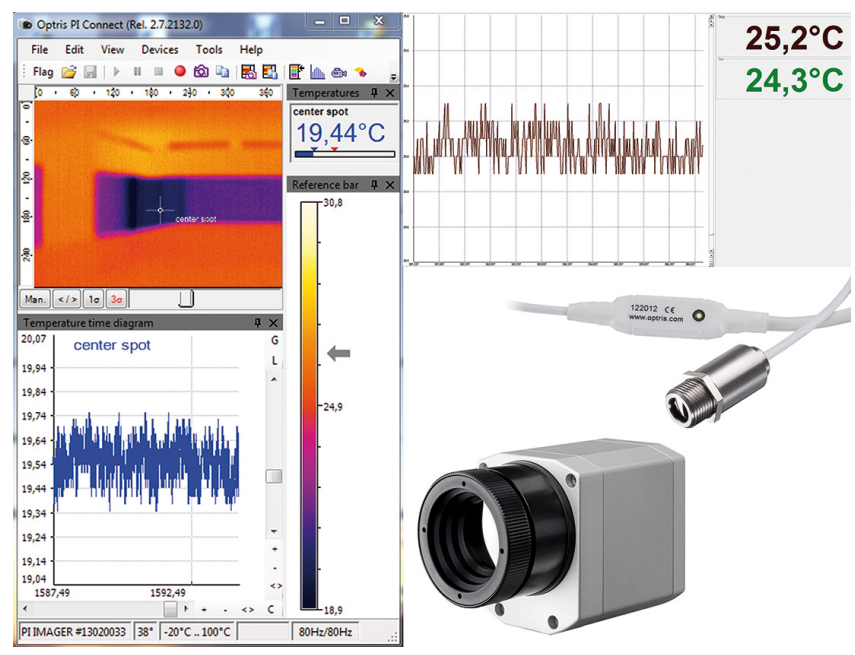

Figure 3. Noise measurements on an Optris PI400 camera with $80 \mathrm{~Hz}$ image frame rate; $\Delta T_{p-p}$ is $0.4 \mathrm{~K}$ and thus $67 \mathrm{mK}$ NETD. The same noise level is obtained when using a CSmicroLT pyrometer with a response time $\left(t_{95}\right)$ of $30 \mathrm{~ms}$.

commonly used estimation allows an effective comparison between the performance parameters of cameras and pyrometers:

$$
D_{/ S \text { meas }}=\frac{1}{3 \mathrm{IFOV}_{\mathrm{geo}}} .
$$

\subsection{Thermal resolution}

Caused by the noise of the IR detector, its preamplification and serialization electronics, IR measuring devices display temperature fluctuations that are normally distributed. The deviation of temperatures around a mean value can be specified as the noise-equivalent temperature difference (NETD). Although rather low values between 10 and $150 \mathrm{mK}$ are listed in the data sheets, according to HAMEG Instruments $\mathrm{GmbH}$ (2013) the observed maximum and minimum differences resulting from the noise distribution are

$$
\Delta T_{p-p}=6 \text { NETD. }
$$

Various noise reduction mechanisms - such as the averaging of image or measurement sequences and measurement time constants that are dependent on signal changes - can be used to stabilize the displayed temperatures. This noise signal still differs significantly from the reproducibility specified in data sheets, which includes additional systematic measurement error resulting from varying environmental conditions. Typically, the reproducibility is double the observed minimum-maximum noise value. With regard to measurement accuracy (which is linked to calibration standards), a further doubling of the error range can be expected: using the radiation thermometer in Fig. 3 for an object at $23^{\circ} \mathrm{C}$ at $67 \mathrm{mK}$ NETD, a reproducibility of $\pm 0.5 \mathrm{~K}$ and an accuracy

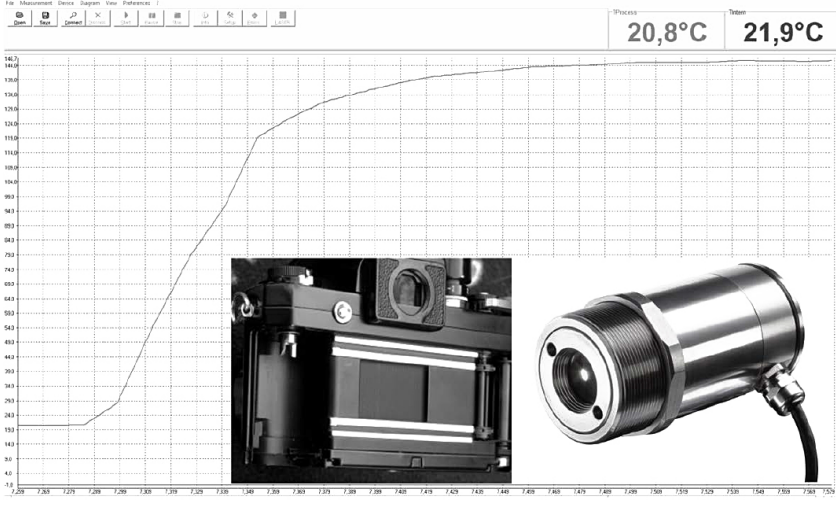

Figure 4. Measuring the response time for a CTlaser LT radiation thermometer using the focal-plane shutter of a traditional camera, according to VDI/VDE standard 3511, Sect. 4.3. (One abscissae interval represents $10 \mathrm{~ms}$; the ordinate is split in $5 \mathrm{~K}$ steps).

of $\pm 1 \mathrm{~K}$ can be attained. Often, a temperature coefficient for the measurement error is specified which describes the accuracy of the thermometer within the entire operating temperature range. The measurement accuracy of cameras is strongly influenced by varying ambient temperatures. A signal drift occurs during the time interval between two offset compensations. This drift can be described with a reproducibility of $\pm 1 \mathrm{~K}$ and measurement accuracy of $\pm 2 \mathrm{~K}$.

\subsection{Time behavior}

The measurement time constant for pyrometers is generally described as the response time ( $\left.t_{95}\right)$ that a radiance proportional signal requires after a signal jump to reach $95 \%$ of its final value ( $S_{\infty}$; Piotrowski and Rogalski, 2013). Thus for the corresponding bandwidth $(\Delta f)$ of the measurement channel,

$\Delta f=\frac{3}{t_{95}}$.

The acquisition time $\left(t_{\mathrm{ex}}\right)$ is a parameter that is independent of any time lag between the input and output signals. It is defined as the minimum amount of time that an object must remain in the field of view to be detected at a defined signal amplitude (of $x$ percent). For imaging devices, the imaging frame rate $\left(f_{\mathrm{B}}\right)$ is an indicator of the time behavior. Due to the sampling theorem the maximum transferable signal bandwidth is limited to half of this value.

However, the acquisition time for short-term events is surprisingly low. Figure 5 shows a hot object, which has been exposed by the focal-plane shutter of a camera for $1 \mathrm{~ms}$. The signal amplitude has decreased to about $8 \%$ of the original value. However, it can be corrected by using an appropriate signal correction method (based on known reference temperatures in the image). After the exposure, three additional images are taken with the same relative image content and decreasing amplitude. In order to estimate the microbolometer's time constant, the assumption can be made that 
a)

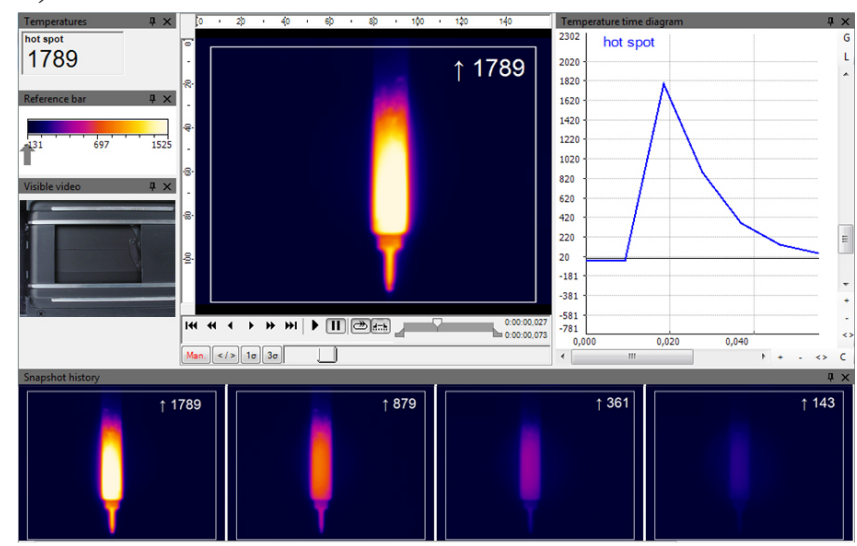

b)

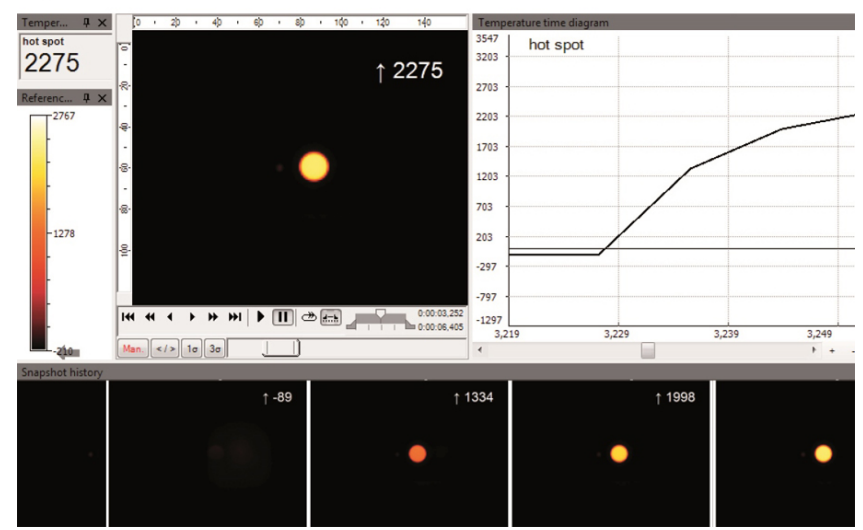

Figure 5. (a) Snapshot representation of a moving soldering iron after a $1 \mathrm{~ms}$ exposure in front of a $120 \mathrm{~Hz}$ frame rate PI160 thermal imaging camera. (b) Time behavior after an instantaneous opening of a photographic focal-plane shutter in front of a black body at $147^{\circ} \mathrm{C}$. Displayed numbers are radiation proportional values similar to signal voltages measured by the video AD converter of the imager.

it exhibits a simple low-pass behavior. The sensor time constant can then be shown as

$t_{63}=\frac{1}{f_{\mathrm{B}}} \ln \left(\frac{S_{\infty}-S_{1}}{S_{\infty}-S_{2}}\right)$.

Here, $S_{1}$ and $S_{2}$ denote the radiance proportional values from two consecutive images. $S_{\infty}$ is the final value which results from the decreasing or increasing signal behavior (as shown in Fig. 5). The calculated bolometer time constant of about $8 \mathrm{~ms}$ corresponds fairly well with the data from Durand et al. (2011). The following detectivity analysis assumes that the imaging devices have a signal bandwidth of

$\Delta f=\frac{1}{t_{63}}$.

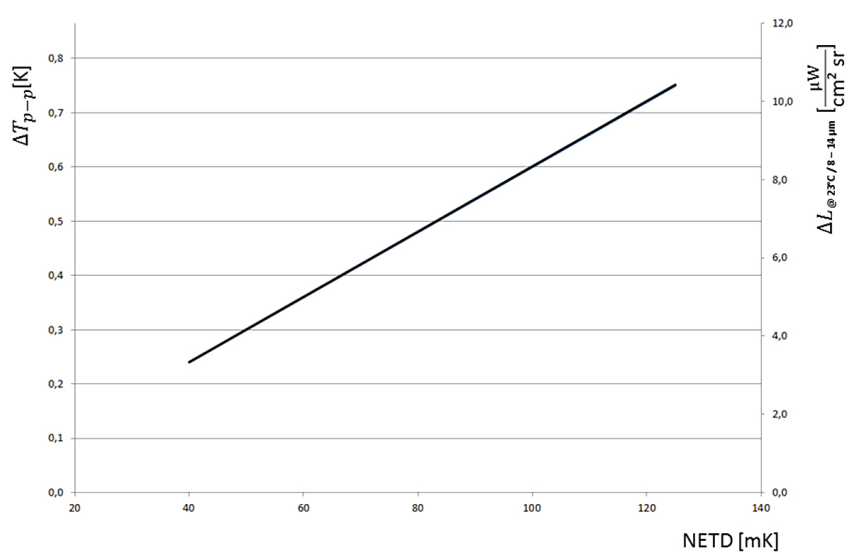

Figure 6. Noise-equivalent radiance difference and peak-to-peak noise as a function of the NETD at $23^{\circ} \mathrm{C}$ in the $8-14 \mu \mathrm{m}$ spectrum.

\subsection{Detectivity}

In order to compare the performance of pyrometers with infrared cameras, it is necessary to analyze the specific detectivity $\left(D^{*}\right)$ as the main sensor parameter as well as the characterization of the optics. The detector and optics are the two most important cost factors.

The detectivity is a measure of the noise-equivalent beam flux (NEP), normalized with respect to area and bandwidth:

$D^{*}=\frac{\sqrt{\Delta f A_{\mathrm{s}}}}{\mathrm{NEP}}$.

A target NETD for the IR measuring device is assumed for the following analyses. According to Planck's radiation law, a surface radiates with a radiance $L$ which is dependent on the spectrum range in use and the surface temperature (Piotrowski and Rogalski, 2013). A radiance difference $(\Delta L)$ is defined which corresponds to the temperature difference NETD at an ambient temperature of $23^{\circ} \mathrm{C}$ (296 K). Thus

$$
\begin{aligned}
\Delta L & =\frac{c 1}{\Omega_{0}}\left(\int_{8 \mu \mathrm{m}}^{14 \mu \mathrm{m}} \lambda^{-5}\left(e^{\frac{c_{2}}{\lambda(296 \mathrm{~K}+\mathrm{NETD})}}-1\right)^{-1} d \lambda\right. \\
& \left.-\int_{8 \mu \mathrm{m}}^{14 \mu \mathrm{m}} \lambda^{-5}\left(e^{\frac{c_{2}}{\lambda(296 \mathrm{~K})}}-1\right)^{-1} d \lambda\right),
\end{aligned}
$$

with $c_{1}=1.191 \times 10^{-16} \mathrm{~W} \mathrm{~m}^{2}$ and $c_{2}=0.01439 \mathrm{~m} \mathrm{~K}$. Figure 6 demonstrates that Eqs. (5) and (10) can be used to calculate the noise-equivalent radiance difference $(\Delta L)$ from a predefined NETD in addition to a max-to-min temperature fluctuation. The NEP can be calculated from the $\Delta L$ via the transmittance $(\tau)$ of the optical system, the sensor surface $\left(A_{\mathrm{S}}\right)$ and the solid angle that the optics have relative to the sensor. According to Bernhard (2004), we can see that the detectivity is determined by the quality of the optics (expressed by the f-number $(F)$, transmittance and the optical diameter $\left.\left(d_{L}\right)\right)$ as well as the temporal, geometric and thermal resolutions: 


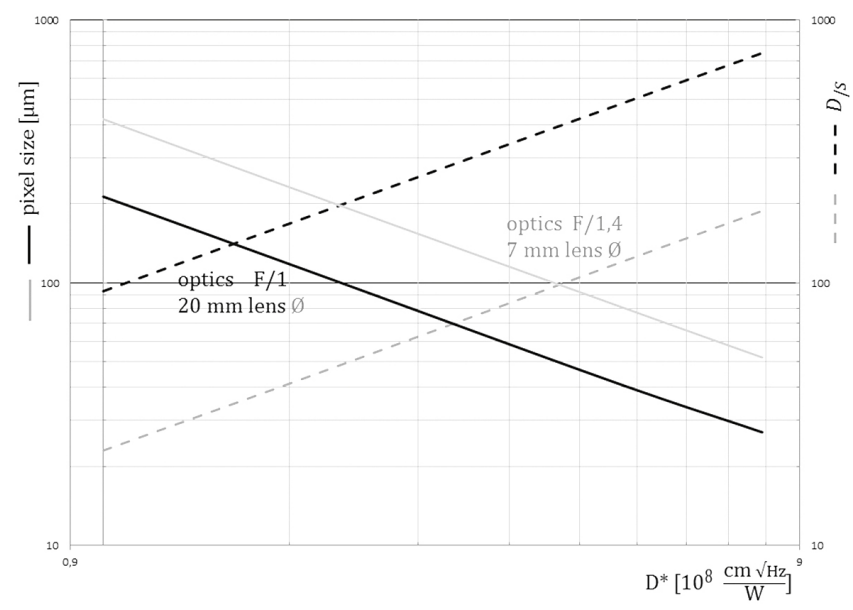

Figure 7. Sensor dimensions and achievable distance ratios as a function of the detectivity level of the thermal sensor.

$D^{*}=\frac{4 F}{\pi \tau d_{L}} \sqrt{\Delta f} \frac{D_{/ S \text { geo }}}{\Delta L}$.

Table 1 lists the interdependent performance parameters for mass-produced IR measuring devices. The resulting detectivity reveals substantial differences between the thermopiles used in the pyrometers and the cameras' microbolometers. Since the f-number of the optics can not be chosen arbitrarily large (due to power limitations) and since the lens diameter is limited by cost and handling restraints, greater distance ratios can only be achieved with a smaller sensor geometry. Thus more powerful sensors with improved detectivity are inevitably designed with smaller sensor dimensions. Figure 7 clarifies this relationship: for a NETD of $70 \mathrm{mK}$, different specific detectivities and various lens performance classes place specific demands on the sensor dimensions associated with the achievable distance ratios. Developers of sensors should always take these relationships into account in order to target, in addition to an increased sensitivity and reduced noise, a geometric miniaturization.

\section{Application examples for industrial cameras}

Such cameras are used in industrial applications to detect defects (such as hot spots in solar facilities) and also for inspecting the uniformity of heating and cooling processes (when using plastic molds, for example). In research and development, the analysis of thermal designs is a key area, as is solving the challenge of dissipating heat away from miniature electronic components and high-power LEDs.

Similar to the trend of cameras replacing pyrometers, the following specific application indicates that imagers may also influence the market of line scanning devices. Often the optical access to the object may be limited, as is the case

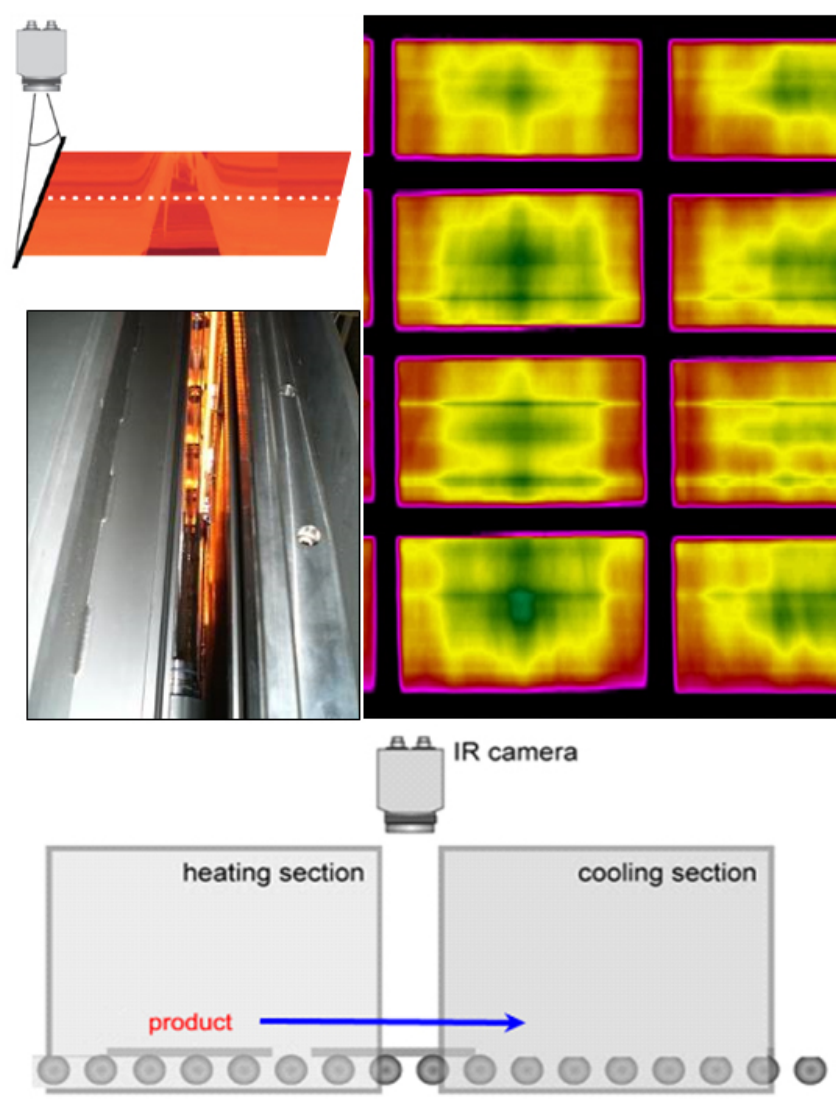

Figure 8. IR cameras used as line scanners allow the object to be sighted through extremely narrow viewing slits. Large-scale measurement images can thus be reconstructed by concatenating the lines of consecutive images.

at the exit of tunnel furnaces. Here the high optical resolution permits the object to be sighted through narrow viewing slits. It is sufficient then to measure the object's temperature in only a few lines per image. Due to the high image frequency (and thus high line frequency) of $120 \mathrm{~Hz}$, it is possible to reconstruct a variably long thermal image of the object in the furnace even when dealing with large material feed rates. The software permits variable image construction, in particular diagonally arranged measurement lines, in which the visual field is $25 \%$ larger relative to the horizontal view. Corresponding digital algorithms correct any barrel-shaped distortions in the optical system. Figure 8 shows an example of the use of thermal imaging cameras for curing glass surfaces. Only a very narrow gap is used for measuring the glass sheets as the $580^{\circ} \mathrm{C}$ heated plates move by $2 \mathrm{~m} \mathrm{~s}^{-1}$ between the heating and cooling sections of the curing facility.

The sheets are measured before they are cooled to inspect their homogeneity and thus evaluate the degree of thermal stress. The thermal images then created show the heat distribution in the furnace and allow the individual heating sections to be controlled more precisely. 
Table 1. A comparison of basic performance parameters of radiation thermometers and IR cameras (at $23^{\circ} \mathrm{C}$ object temperature, $8-14 \mu \mathrm{m}$ range).

\begin{tabular}{|c|c|c|c|c|}
\hline \multirow[t]{2}{*}{ Parameter } & & \multicolumn{2}{|c|}{ Radiation thermometer } & \multirow{2}{*}{$\begin{array}{l}\text { IR camera } \\
\text { Optris PI400 }\end{array}$} \\
\hline & & Optris CTLT & Optris LS & \\
\hline \multirow[t]{4}{*}{ Thermal } & NETD [mK] & 67 & 40 & 67 \\
\hline & $\Delta T_{p-p}[\mathrm{~K}]$ & 0.40 & 0.24 & 0.40 \\
\hline & Reproducibility $\left[{ }^{\circ} \mathrm{C}\right]$ & \pm 0.5 & \pm 0.5 & \pm 1 \\
\hline & Accuracy $\left[{ }^{\circ} \mathrm{C}\right]$ & \pm 1 & \pm 0.75 & \pm 2 \\
\hline \multirow[t]{5}{*}{ Geometrical } & $D_{/ \text {Sgeo }}$ & 15 & 75 & 560 \\
\hline & $D_{/ S \text { meas }}$ & 15 & 75 & 187 \\
\hline & $\mathrm{IFOV}_{\text {geo }}[\mathrm{mrad}]$ & 66.6 & 13.3 & 1.8 \\
\hline & FOV $\left[^{\circ}\right]$ & 3.8 & 0.8 & 39 \\
\hline & Pixel $p_{\#}$ & 1 & 1 & 382 \\
\hline \multirow[t]{2}{*}{ Temporal } & $t_{95}[\mathrm{~ms}]$ & 30 & 120 & 25 \\
\hline & $\Delta f / f_{\mathrm{B}}[\mathrm{Hz}]$ & 100 & 25 & 120 \\
\hline \multirow[t]{3}{*}{ Optics } & f-number $F$ & 1.50 & 1.60 & 0.80 \\
\hline & Aperture $d_{L}[\mathrm{~cm}]$ & 0.8 & 2.1 & 1.8 \\
\hline & Transmission $\tau$ & 0.7 & 0.6 & 0.75 \\
\hline \multirow[t]{2}{*}{ Detector } & Sensor area $A_{\mathrm{s}}\left[\mu \mathrm{m}^{2}\right]$ & $\varnothing 750$ & $\varnothing 450$ & $25 \times 25$ \\
\hline & Detectivity $\left[\frac{\mathrm{cm} \sqrt{\mathrm{Hz}}}{W}\right]$ & $1.1 \times 10^{8}$ & $2.0 \times 10^{8}$ & $8.5 \times 10^{8}$ \\
\hline
\end{tabular}

\section{The outlook}

The calculation methods presented here accurately describe measurements obtained from series products. Smaller sensors with higher sensitivity will continue to influence the key parameters for one-dimensional and two-dimensional noncontact radiation thermometers. The significantly improved performance of bolometers compared to thermopiles will open up new application fields in which small measuring points will be measured with variable positioning and small thermal contrast over large relative distances. Ultimately it will be the cost of such devices that plays the greatest role in widening or limiting their use.

\section{Brief biography}

Dr. Ulrich Kienitz is the General Manager of Optris GmbH, one of Germany's leading companies in the field of noncontact temperature measurement technology. Previously he managed the European division for a major American IR instrumentation company for over 10 years. As the holder of numerous patents, he has significantly influenced the design of many of the major infrared thermometry devices on the market.

Edited by: G. S. Aluri

Reviewed by: two anonymous referees 


\section{References}

Bernhard, F.: Technische Temperaturmessung, Physikalische und meßtechnische Grundlagen, Sensoren und Meßverfahren, Meßfehler und Kalibrierung, Berlin, Heidelberg: SpringerVerlag, 2004.

Datasheet Heimann Sensor GmbH: Miniature Thermopile Sensors for Remote Temperature Measurement and Gas Analysis, available at: http://www.heimannsensor.com/Datasheet-2b-TO18_ rev2.pdf, last access: 19 November 2013.

Datasheet Micro-Hybrid Electronic GmbH: One Channel Thermopile Detector TS1x80B-A-D0.48, available at: http: //www.micro-hybrid.de/fileadmin/user/IR-systems-documents/ Datenblaetter/Thermopiles/MH_TS1x80B-A-D0.48.pdf last access: 19 November 2013.

Durand, A., Tissot, J. L., Robert, P., Cortial, S., Roman, C., Vilain, M., and Legras, O.: VGA $17 \mu \mathrm{m}$ development for compact, low power systems, available at: http://www.ulis-ir.com/uploads/ Documents/8012-43_ULIS.pdf (last access: 8 November 2013), 2011.

FLIR Corporation - INVESTOR PRESENTATION, March 2013 forward looking, available at: http://www.flir.com/investor/, last access: 15 March 2013.
HAMEG Instruments GmbH: Professional Article, What is noise?, available at: http://www.hameg.com/articles.0.html?\&no_ cache $=1 \& \mathrm{~L}=1 \& \mathrm{tx} \_$mdownloads_pi1[mode] =download $\&$ tx hmdownloads_pi1[uid]=3046, last access: 19 November 2013.

Piotrowski, J. and Rogalski, A.: High-Operating-Temperature Infrared Photodetectors, available at: http://spie.org/samples/ PM169.pdf, last access: 19 November 2013.

Product brochure Optris GmbH: Infrared cameras, The most portable infrared online camera, Version D2013-06-A, available at: http://www.optris.com/downloads-infrared-cameras?file=tl_ files/pdf/Downloads/Infrared20Cameras/PI_Brochure.pdf, last access: 19 November 2013.

VDI/VDE 3511, Sheet 4.3: Radiation Thermometry/Calibration of Radiation Thermometers, Edn. Dec., 2004.

VDI/VDE 3511, Sheet 4.4: Anwendung der Thermografie zur Diagnose in der Instandhaltung, Edn. June, 2010.

YOLE Developement: Uncooled Infrared Cameras and Detectors; Report, Lyon, 2010. 